\title{
SOX2 regulates self-renewal and tumorigenicity of stem-like cells of head and neck squamous cell carcinoma
}

\author{
S H Lee ${ }^{1}$, S-Y Oh ${ }^{2}$, S I Do ${ }^{3}, \mathrm{H}$ J Lee ${ }^{3}, \mathrm{H} \mathrm{J} \mathrm{Kang}^{4}$, Y S Rho ${ }^{5}$, W J Bae ${ }^{6}$ and Y C Lim ${ }^{*} 4$ \\ ${ }^{1}$ Department of Otorhinolaryngology-Head and Neck Surgery, Kangbuk Samsung Hospital, Sungkyunkwan University School of \\ Medicine, Seoul, Korea; ${ }^{2}$ School of Life Sciences and Biotechnology, Korea University, Seoul, Korea; ${ }^{3}$ Department of Pathology, \\ Kangbuk Samsung Hospital, Sungkyunkwan University School of Medicine, Seoul, Korea; ${ }^{4}$ Department of Otorhinolaryngology- \\ Head and Neck Surgery, Research Institute of Medical Science, Konkuk University School of Medicine, Seoul, Korea; ${ }^{5}$ Department \\ of Otorhinolaryngology-Head and Neck Surgery, Ewha University School of Medicine, Seoul, Korea and ${ }^{6}$ Department of \\ Otolaryngology-Head and Neck Surgery, Hallym University College of Medicine, Seoul, Korea
}

Background: Head and neck squamous cell carcinomas (HNSCCs) display cellular heterogeneity and contain cancer stem cells (CSCs). Sex-determining region Y [SRY]-box (SOX)2 is an important regulator of embryonic stem cell fate and is aberrantly expressed in several types of human tumours. Nonetheless, the role of SOX2 in HNSCC remains unclear.

Methods: We created cells ectopically expressing SOX2 from previously established HNSCC cells and examined the cell proliferation, self-renewal capacity, and chemoresistance of these cells compared with control cells. In addition, we knocked down SOX2 in primary spheres obtained from HNSCC tumour tissue and assessed the attenuation of stemness-associated traits in these cells in vitro and in vivo. Furthermore, we examined the clinical relevance of SOX2 expression in HNSCC patients.

Results: SOX2 is aberrantly expressed in primary tissue of HNSCC patients but not in healthy tissue. SOX2 expression correlated with tumour recurrence and poor prognosis of HNSCC patients. Ectopic expression of SOX2 induced cell proliferation via cyclin B1 expression and stemness-associated features, such as self-renewal and chemoresistance. In addition, a knockdown of SOX2 in HNSCC CSCs attenuated their self-renewal capacity, chemoresistance (through ABCG2 suppression), invasion capacity (via snail downregulation), and in vivo tumorigenicity.

Conclusions: These results suggest that SOX2 may have important roles in the 'stemness' and progression of HNSCC. Targeting SOX2-positive tumour cells (CSCs) could be a new therapeutic strategy in HNSCCs.

Head and neck squamous cell carcinoma (HNSCC) is the sixth most common cancer, with an incidence rate of more than half a million new cases each year worldwide (Jemal et al, 2003). They arise from diverse anatomical subsites, including the oral cavity, pharynx, and larynx. Despite advances in treatment modalities, survival rates have not improved significantly in $>30$ years, with a $50 \%$ 5-year survival rate at present (Carvalho et al, 2005). Unchangeable mortality from this cancer may be due to a high incidence of therapy-resistant local and regional recurrence and distant metastases (Yamano et al, 2010). An improvement in the survival of patients with HNSCC will thus require a deeper understanding of the biology of this cancer.

HNSCC displays cellular heterogeneity and contains cancer stem cells (CSCs), functionally defined by extensive self-renewal capacity, a differentiation ability, and potential xenograft tumorigenicity (Lim et al, 2011). Although CSCs still need to be fully characterised, recent progress in the notion of HNSCC CSCs has provided a deeper understanding about the mechanism of 
increased radio- and chemoresistance, resulting in tumour recurrence and metastatic spread: common causes of death in the majority of patients with HNSCC (Albers et al, 2012). Therefore, identification of regulators that control the tumorigenic potential of HNSCC CSCs might pave the way for new therapeutic strategies against these cancer stem cells to improve HNSCC treatment.

SOX (sex-determining region Y [SRY]-box) family transcription factors are well-established regulators of cell fate during development (Sarkar and Hochedlinger, 2013). To date, 20 diverse SOX genes have been discovered in mice and humans (Sarkar and Hochedlinger, 2013). Of these, SOX2 is crucial for the derivation of embryonic stem cells (ESCs) from the inner cell mass and for the maintenance of ESCs themselves (Keramari et al, 2010). In addition, SOX2 is a marker of stem and progenitor cells in diverse adult tissues, including squamous epithelia of the tongue (Okubo et al, 2009). Recently, SOX2 was found to act as an oncogene in some epithelial cancers (Chen et al, 2012; Leis et al, 2012). Amplification of the SOX2 locus was identified in human SCC of the lung (23\%) and oesophagus (15\%; Bass et al, 2009). A knockdown of SOX2 decreases anchorage-independent growth in SOX2-amplified lung SCC cell lines (Bass et al, 2009). Some authors suggested that SOX2 expression promotes invasiveness of tumour cells in glioma (Ikushima et al, 2009). In addition, SOX2 expression is closely associated with poor prognosis in patients with HNSCC (Du et al, 2011; Schröck et al, 2013; Tang et al, 2013; Schröck et al, 2014). In light of these findings, SOX2 may be linked with stemness of cells of diverse solid tumours, but there have been no thorough studies of SOX2 in HNSCC. In the current study, we assessed the role of SOX2 in the maintenance of the stem-like phenotype in the cells of HNSCC. We also evaluated SOX2's potential as a therapeutic target for the elimination of HNSCC CSCs as the main instigators of this disease.

\section{MATERIALS AND METHODS}

Cell culture and reagents. HNSCC cell lines SNU1041 and FaDu were obtained from Korea Cell Line Bank (Seoul, Korea) and maintained in Dulbecco's Modified Eagle's Medium (DMEM; Invitrogen, Carlsbad, CA, USA), 10\% FBS (Invitrogen), and penicillin/streptomycin (Invitrogen). Primary sphere cells (K3, $\mathrm{K} 4$, and K5) were isolated from surgical specimens from HNSCC patients, and the CSC properties were validated using a number of functional assays testing the self-renewal capability, stem cell marker expression, chemoresistance, and in vivo tumorigenicity, as reported previously (Lim et al, 2011). Primary sphere cells expanded in serum-free DMEM Ham's F-12 (DMEM/F12) medium supplemented with human recombinant basic fibroblast growth factor (bFGF; $10 \mathrm{ng} / \mathrm{ml}$; R\&D Systems, Minneapolis, MN, USA), N2 supplement (GIBCO, Franklin Lakes, NJ, USA), and epidermal growth factor (EGF; $10 \mathrm{ng} \mathrm{ml}^{-1}$; R\&D Systems). We purchased primary antibodies against proteins Oct4, Nanog, CD44, Snail, cyclin B1, vimentin, and ABCG2 from Santa Cruz Biotechnology (Santa Cruz, CA, USA), Ki-67 from Chemicon International Inc. (Temecula, CA, USA), SOX2 from Abcam (Cambridge, UK), E-cadherin from Cell Signalling Technology (Beverly, MA, USA), and secondary antibodies, anti-rabbit IgG and anti-mouse IgG, from Jackson ImmunoResearch Laboratories (West Grove, PA, USA).

Transwell chamber invasion assay. Transwell chambers (SPL, Seoul, Korea) were used to assess cellular invasiveness. Matrigel was applied to polycarbonate membrane filters with a pore size of $8 \mu \mathrm{m}$. The cells were seeded in the upper part of a Transwell chamber at a density of $10^{5}$ per well in $100 \mu$ l of a serum-free medium. The bottom chamber contained a standard medium supplemented with EGF + bFGF. The chamber was then incubated in $5 \% \mathrm{CO}_{2}$ at $37^{\circ} \mathrm{C}$ for $24 \mathrm{~h}$. The filter in the upper well was removed. Finally, the attached cells in the lower section were stained with crystal violet and intensity was calculated using a spectrophotometer, as described previously (Lai et al, 2005).

Full Materials and Methods are available in the Supplementary Methods.

\section{RESULTS}

SOX2 expression levels correlate with survival of patients with HNSCC. We assessed the relationship between SOX2 expression and the clinicopathological parameters of 69 patients with histologically confirmed HNSCC, as well as the characteristics of 10 cases of healthy head and neck tissues (tonsil tissues), using immunohistochemical staining of paraffin-embedded sections. Demographics of the 69 patients enrolled in this study are presented in Supplementary Table S1A. Immunoreactivity for SOX2 was detected in 31 of 69 cases (45\%) but not in healthy tonsil tissues. Of 31 tumour tissues with SOX2 staining, there were eight (26\%) with strong SOX2 expression, nine (29\%) with moderate expression, and 14 (45\%) with weak expression (Figure 1A). SOX2 expression was regrouped as low (negative or weak, $n=52$ ) or high (moderate or strong, $n=17$ ) for statistical analysis. Univariate and multivariate analyses revealed that tumours with high positive expression for SOX2 were significantly associated with a recurrence event, with a 4.7 -fold higher risk, compared with tumours with low expression for SOX2 (Supplementary Table S1B). In addition, patients with SOX2-low tumours had a better disease-specific survival rate $(P=0.034)$ compared with patients with SOX2-high tumours, as assessed by means of Kaplan-Meier survival analysis and the log-rank test (Figure 1B). There was no significant correlation between the primary tumour site and SOX2 expression in this cohort (18 oral cavity cancer, 14 oropharynx cancer, 16 larynx cancer, 13 hypopharynx cancer, and 8 others). Because CD44 was reported to be a putative marker for the identification and isolation of HNSCC CSCs, we next performed immunofluorescent assays for SOX2 and CD44 on frozen sections of surgical specimens $(n=5)$. SOX2 could coexist in HNSCC cells expressing the HNSCC CSC marker CD44, suggesting that SOX2 is preferentially expressed in CSCs in HNSCC (Figure 1C and Supplementary Table S2).

SOX2 promotes proliferation of HNSCC cells via cyclin B1 upregulation. To test whether SOX2 enhances tumour proliferation, we examined in vitro growth effects in response to overexpression of SOX2 in two established HNSCC cell lines: SNU1041 and FaDu. The expression level of SOX2 in stably transfected HNSCC cells was confirmed using western blot analysis (Figure 2A). SNU1041-SOX2 and FaDu-SOX2 cells grew more rapidly compared with SNU1041-Neo and FaDu-Neo control cells by day 7 after plating (Figure 2B). The increased growth rates associated with SOX2 overexpression prompted us to analyse cell cycle-regulatory proteins. The results showed a remarkable increase in the transcriptional and translational level of cyclin B1 (Figure 2C and D). To test the relationship between SOX2 and cyclin B1 in relation to cellular proliferation, we downregulated cyclin B1 while SOX2 was overexpressed (Figure 2E). The results showed that the enhancement of proliferation by SOX2 was reversed by transient suppression of cyclin $\mathrm{B} 1$ by means of small interfering RNA (siRNA; Figure 2F). Collectively, these data suggest that proliferation of HNSCC cells can be accelerated by cyclin B1 overexpression, which is caused by overexpression of SOX2.

SOX2 enhances stem cell traits of HNSCC in vitro. Our previous study suggested that a cancer stem cell line from an HNSCC 
A

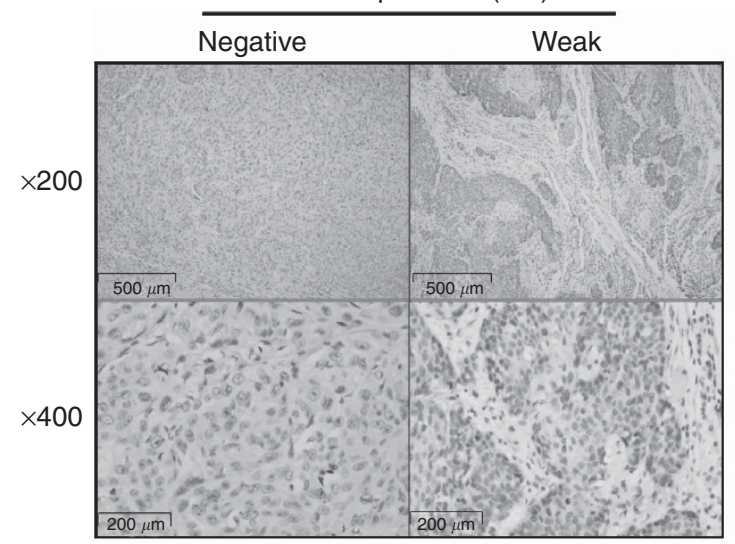

SOX2 expression (high)

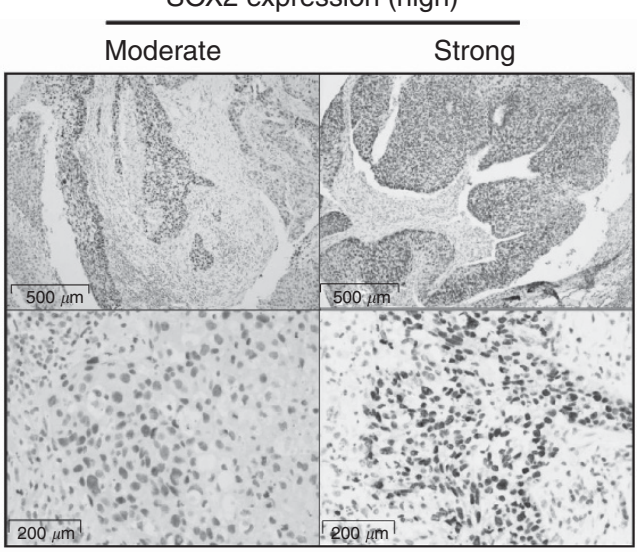

B

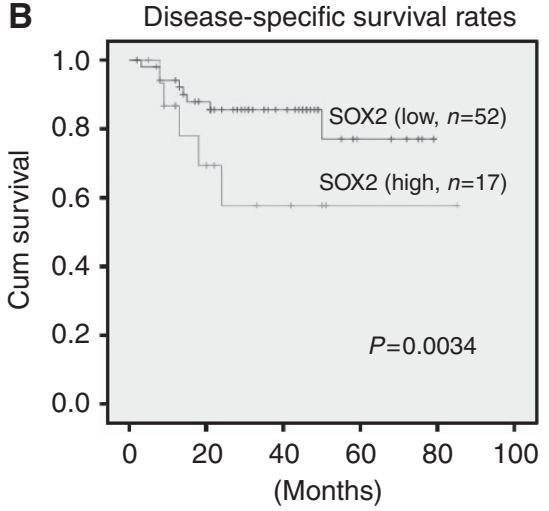

C

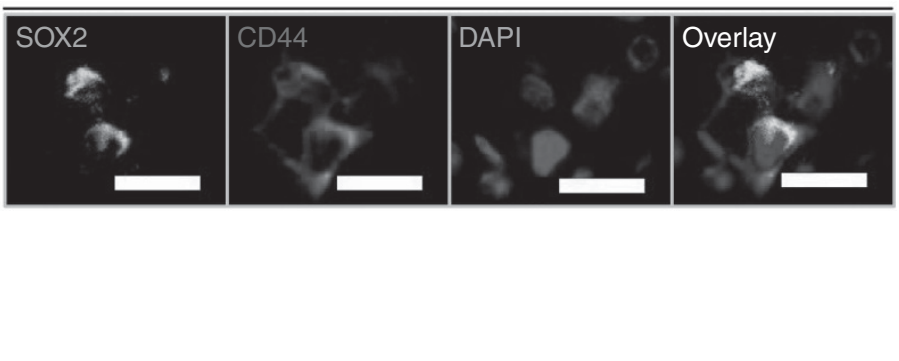

Figure 1. SOX2 expression in primary HNSCC specimens. (A) Immunohistochemical analysis of expression of SOX2 from primary HNSCC specimens (magnification: $\times 200$ and $\times$ 400). (B) The disease-specific survival rate according to SOX2 expression using the Kaplan survival curve. (C) Immunofluorescent images showing colocalization of SOX2 (green) with CD44 (red) on an HNSCC specimen. Nuclei were stained with DAPI (blue). Bar, $20 \mu \mathrm{m}$. A full colour version of this figure is available at the British Journal of Cancer journal online.

patient maintains its properties and expression levels of stem cell factors, but these properties are inhibited when this cell line is exposed to conditions conducive to cell differentiation-for example, culture media that contain serum (Lim et al, 2011). Among those stem cell factors, SOX2 is downregulated after cell culture in a serum-containing medium during the process of loss of stemness properties by the cells. To determine whether SOX2 governs stemness properties in vitro, we first examined changes in OCT4 and NANOG expression while SOX2 was overexpressed in tumour cells (Figure 3A). We found that OCT4 and NANOG were positively regulated by SOX2. Moreover, SOX2 overexpression caused SNU1041 cells to show accelerated tumour sphere formation-a hallmark of self-renewal properties (Figure $3 \mathrm{~B}$ ) when grown in a serum-free medium supplemented with EGF and bFGF; these are reliable suspension culture conditions for healthy stem cells to form spheres and maintain their stemness (Zhang et al, 2008). Similar to previous data that SOX2-positive tumour cells coexpress the stemness marker CD44 (Figure 1C), we performed FACS analysis to measure the number of CD44-positive cells among HNSCC-SOX2 cells compared with control cells (Figure 3C). The data show that overexpression of SOX2 in HNSCC cells increased the CD44-positive population and can explain why the stemness properties can be maintained by SOX2.

SOX2 is highly expressed in HNSCC CSCs. In our previous report, we successfully isolated CSCs from primary HNSCC tissue and pointed out that their specific features are closely related to other types of CSCs (Lim et al, 2011). To elucidate the biological function of endogenous SOX2 in HNSCC CSCs, not ectopic SOX2, we used three primary sphere cell clones (K3, K4, and K5) enriched from primary HNSCC tissue for further experiments. Aldehyde dehydrogenase (ALDH) is thought to be a marker of HNSCC CSCs (Yu et al, 2013). Thus, we first measured ALDH levels using FACS and classified the cells into ALDH ${ }^{\text {high }}$ and ALDH ${ }^{\text {low }}$ subpopulations based on their ALDH expression level, and then measured the SOX2 mRNA level using RT-qPCR in these three cell clones. The results showed that SOX2 expression in ALDH ${ }^{\text {high }}$ cells was significantly higher than in ALDH ${ }^{\text {low }}$ cells (Figure 4A). Next, we generated HNSCC CSCs-shSOX2 cells using lentivirus-mediated shRNA targeting SOX2 to examine the loss of function of SOX2 in HNSCC CSCs. When SOX2 was downregulated in HNSCC CSCs, we found that OCT4 and NANOG, known as putative stemnessassociated factors, were also downregulated (Figure 4B). In addition, the sphere-forming capacity in serum-free conditions, assumed to correspond to self-renewal capacity in vitro, was significantly reduced in HNSCC CSC-shSOX2 cells compared with control cells (Figure 4C). Furthermore, depletion of SOX2 in HNSCC CSCs decreased the percentage of $\mathrm{CD}_{4}{ }^{+}$cells (Figure $4 \mathrm{D}$ ). Taken together, these data suggest that SOX2 is one of the main regulators of CSC features in HNSCC.

SOX2 enhances chemoresistance of HNSCC CSCs through ABCG2 expression. The notion of CSCs received much attention because this hypothesis can explain tumour recurrence and the resistance to traditional anticancer treatments. To verify the possible correlation between SOX2 expression and antitumour drug resistance, we used cisplatin, one of the most popular drugs for the treatment of patients with HNSCC, for incubation with 

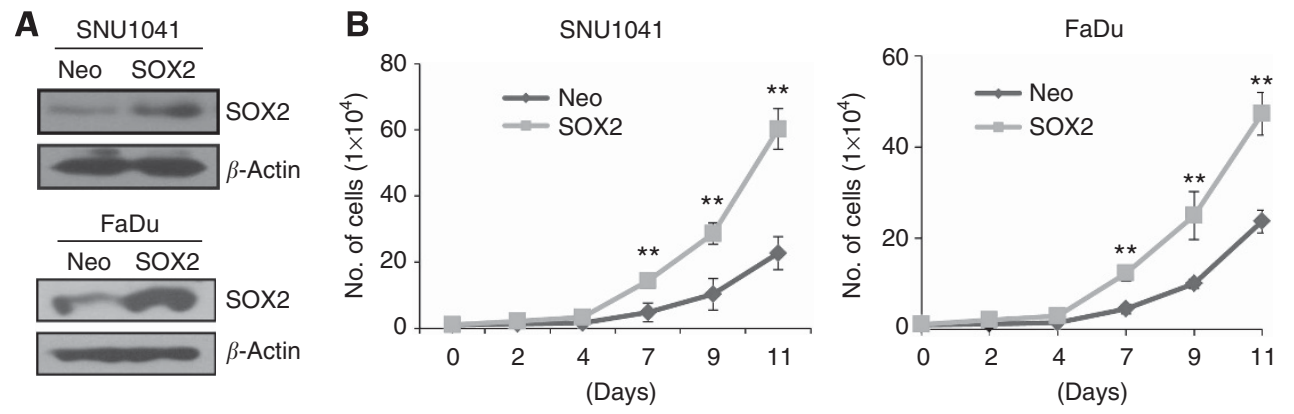

C

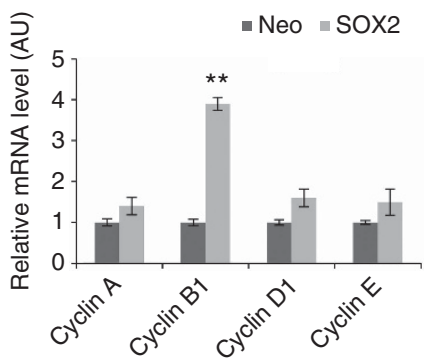

D

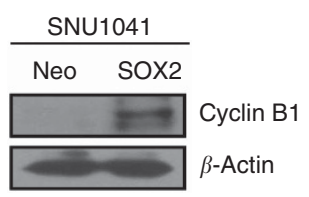

$\mathbf{E}$

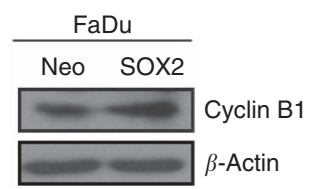

SNU1041-SOX2 Scramble siCyclin B1

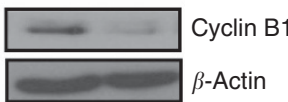

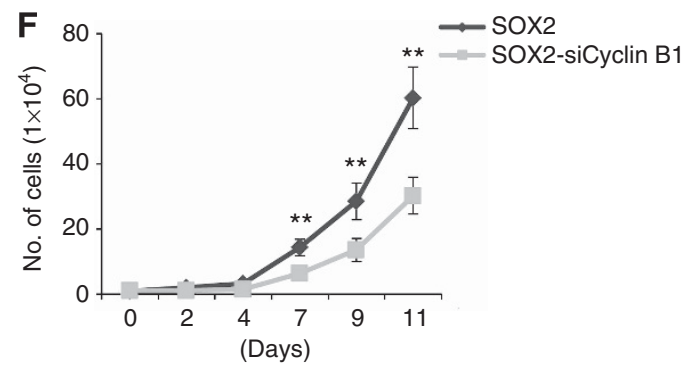

Figure 2. Cell proliferation induced by SOX2 overexpression via upregulation of cyclin B in vitro. (A) Expression of SOX2 in control cells and in SOX2-overexpressing SNU1041 and FaDu cells analysed using western blotting. (B) The in vitro growth rate of control and SOX2-overexpressing SNU1041 and FaDu cells $(n=3)$. ${ }^{* \star} P<0.01$. After cells $\left(2 \times 10^{4}\right)$ were plated and grown in media, the total number of cells was counted every 2 days. (C) Expression of cyclin A, cyclin B1, cyclin D1, and cyclin E mRNAs in control and in SOX2-overexpressing cells was determined by means of quantitative RT-PCR. ${ }^{*} P<0.01$. (D) Induced protein levels of Cyclin B1 in SOX2-overexpressing SNU1041 and FaDu cells according to western blot analysis. (E) The suppressed expression of Cyclin B1 in SOX2-overexpressing SNU1041 cells that were transiently transfected with siCyclin B1, confirmed by western blot analysis. (F) The in vitro growth rate of SOX2-overexpressing SNU1041 cells that were transiently transfected with either scrambled siRNA or siCyclin B1 $(n=3)$. ${ }^{\star \star} P<0.01$.

A

\begin{tabular}{|c|c|}
\hline SNU1041 & \\
\hline Neo SOX2 & \\
\hline 20 & Oct4 \\
\hline-1 & Nano \\
\hline 2 & $\beta$-Acti \\
\hline
\end{tabular}

B

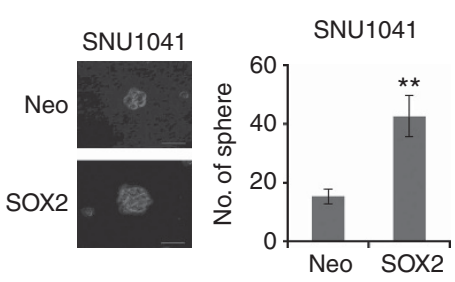

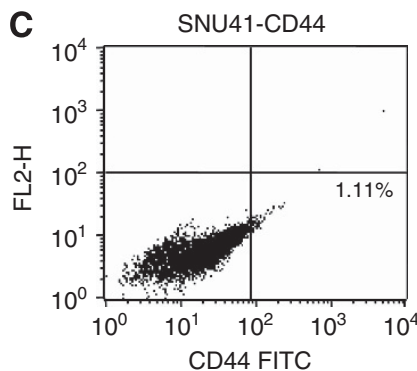

CD44 FITC

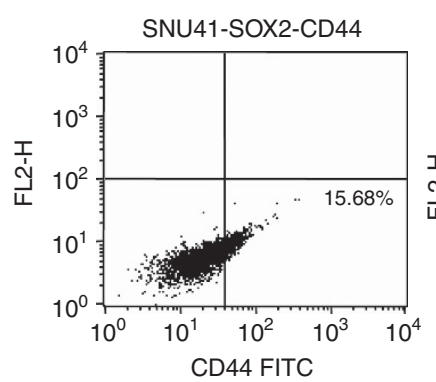

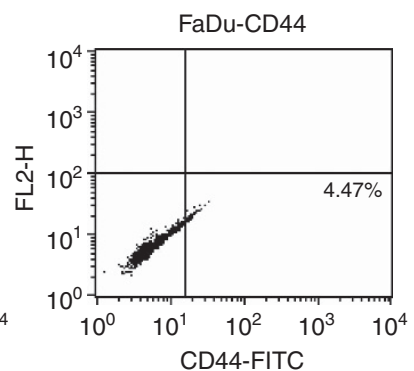

CD44-FITC

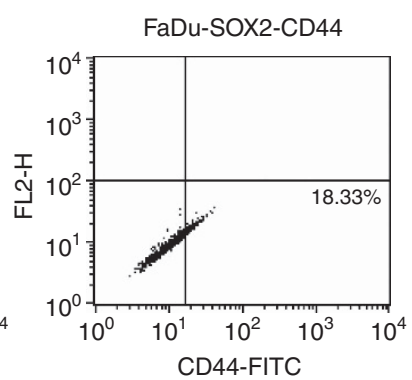

Figure 3. SOX2 induces stem cell properties in HNSCC cells in vitro. (A) Expression of induced stemness-associated factors - OCT4 and NANOG - determined by western blot analysis. (B) Representative photos showing the morphology of spheres grown in serum-free cultures (left). The number of neurospheres $(>10 \mu \mathrm{m})$ generated from control and SOX2-overexpressing SNU1041 cells grown in a serum-free culture medium for 14 days (right); scale bar, $10 \mu \mathrm{m}$. Cells were seeded in a 24-well plate at a density of 200 cells per well and cultured in serum-free media with EGF and bFGF commitment every second day. Spheres with a diameter exceeding $10 \mu \mathrm{m}$ were counted after 14 days. (C) Flow cytometric analysis showed a moderate increase in CD44-positive population in control cells and in SOX2-overexpressing SNU1041 cells. IgG was used as a negative control. Cells were dissociated into a single-cell suspension, labelled with an anti-CD44 primary antibody, and then subjected to flow cytometric analysis on a FACSCalibur instrument.

control and SNU1041-SOX2 cells. We measured tumour cell viability in a dose-dependent manner. The results show that SOX2 increased the number of cisplatin-resistant tumour cells, suggesting that SOX2-mediated chemoresistance to cisplatin is partially attributable to the increase in the CSC subpopulation (Figure 5A). To extend these experiments to CSCs, we used SOX2- 
A K3

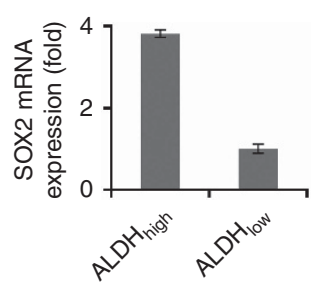

C

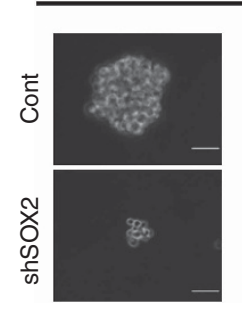

K4

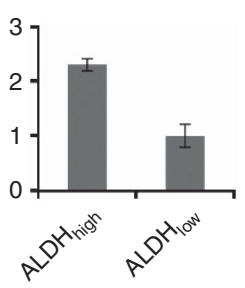

K3

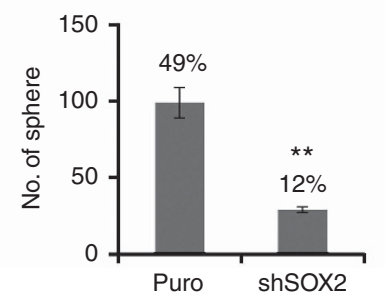

K5

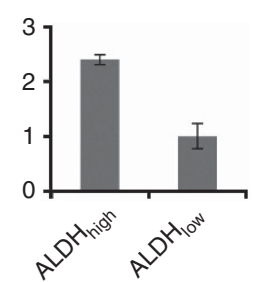

B $\mathrm{K} 3$

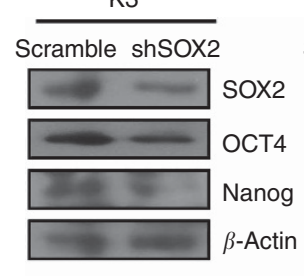

K4

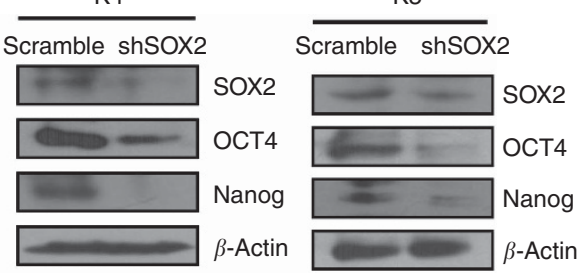

K4

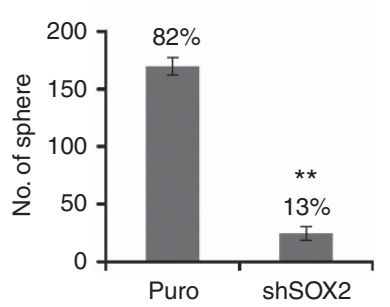

K5

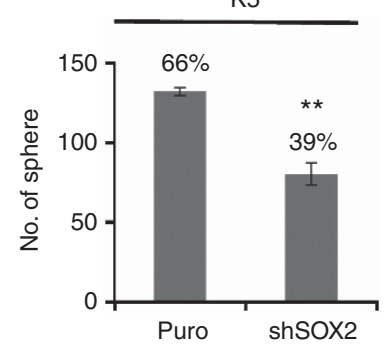

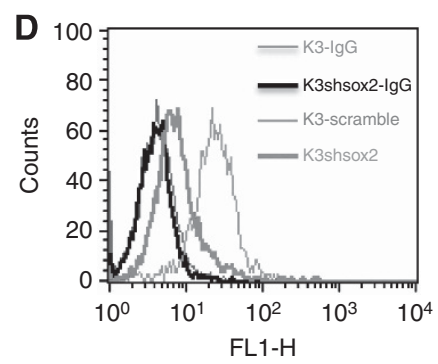
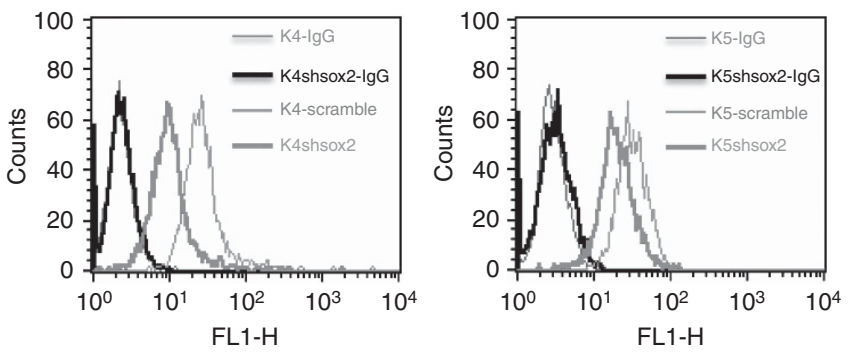

Figure 4. SOX2 controls the stemness properties of primary HNSCC stem-like cells. (A) Quantitative PCR analysis showing that the ALDH ${ }^{\text {high }}$ population in HNSCC stem-like cells exhibits a relatively high expression level of SOX2 mRNA compared with ALDH ${ }^{\text {low }}$ cells. These HNSCC stemlike cells were cultured in serum-free media with EGF and bFGF. (B) Western blot analysis of protein levels of OCT4 and NANOG in HNSCC stemlike cells transfected with scrambled shRNA and in HNSCC stem-like cells transfected with shSOX2. (C) Representative photos showing the morphology of spheres in HNSCC stem-like cells transfected with scrambled shRNA and in HNSCC stem-like cells transfected with shSOX2 (left). The number of neurospheres $(>10 \mu \mathrm{m})$ generated from HNSCC stem-like cells transfected with scrambled shRNA and from HNSCC stem-like cells transfected with shSOX2 for 14 days (right). Cells were seeded in a 24-well plate at a density of 200 cells per well and cultured in serum-free media with EGF and bFGF commitment every second day. (D) Flow cytometric analysis showing a decrease in the CD44-positive population in HNSCC stem-like cells transfected with scrambled shRNA and in HNSCC stem-like cells transfected with shSOX2. IgG was used as a negative control.

deficient primary sphere cells and confirmed their resistance to cisplatin treatment by measuring cell viability (Figure $5 \mathrm{~B}$ and Supplementary Figure S1). These data proved that cell viability after cisplatin treatment is decreased by SOX2 downregulation and suggest that SOX2-mediated drug resistance also applies to CSCs. A possible mechanism of the chemoresistance of CSCs is the increased ability to exclude exogenous and endogenous toxic substances by means of the ATP-binding cassette (ABC) transporters (Chen and Sikic, 2012). Among various $A B C$ transporters, ABCG2 is thought to have a critical role in HNSCC CSC (Lim et al, 2011). Thus, we quantified ABCG2 expression in HNSCC CSC-shSOX2 cells and found that ABCG2 was weakly expressed in SOX2-deficient CSCs compared with control cells (Figure 5C). In addition, downregulation of ABCG2 in HNSCC CSCs restored drug sensitivity after cisplatin treatment in HNSCC CSCs (Figure 5D and E). All these data suggest that SOX2 has a major role in drug resistance of HNSCC CSCs.

SOX2 increases the invasiveness of HNSCC CSCs through epithelial mesenchymal modulation. Tumour recurrence in patients is related not only to drug resistance of tumour cells (to survive chemotherapy) but also to invasiveness, which helps tumour cells to travel to a distant site and to settle there (Howell and Grandis, 2005). To analyse the relation of invasiveness and SOX2, we performed a Transwell assay to measure the invasive ability after downregulation of SOX2 in HNSCC CSCs. The number of cells that drop on the bottom plate was decreased in shSOX2-transfected (knockdown) cells, and downregulation of SOX2 can inhibit invasiveness of HNSCC CSCs (Figure 6A). Because epithelial-mesenchymal transition (EMT) is known to be associated with tumour invasiveness and stem-like traits (Mani et al, 2008), we performed a western blot to assess the change in expression levels of E-cadherin and vimentin: key markers of EMT. We found that SOX2 downregulation induced E-cadherin expression and decreased vimentin expression (Figure 6B). In addition, we screened EMT mediators such as TWIST, SNAIL, and SLUG by means of RT-qPCR (Figure 6C) and found that SNAIL is downregulated when SOX2 is knocked down in HNSCC CSCs (Figure 6D). Because downregulation of SNAIL using siRNA in HNSCC CSCs decreases invasiveness according to the Transwell assay (Figure 6E and $\mathrm{F}$ ), these data suggest that $\mathrm{SOX} 2$ controls tumour invasiveness in HNSCC CSCs via positive regulation of SNAIL expression.

A knockdown of SOX2 inhibits xenografted tumour growth in vivo. To validate the oncogenic properties of SOX2 in vivo, we subcutaneously injected SOX2-deficient HNSCC CSCs into immunocompromised mice and compared their tumorigenic potential with control cells. We found that HNSCC CSC-shSOX2 cells displayed a marked decrease in tumorigenesis (Figure 7A). 

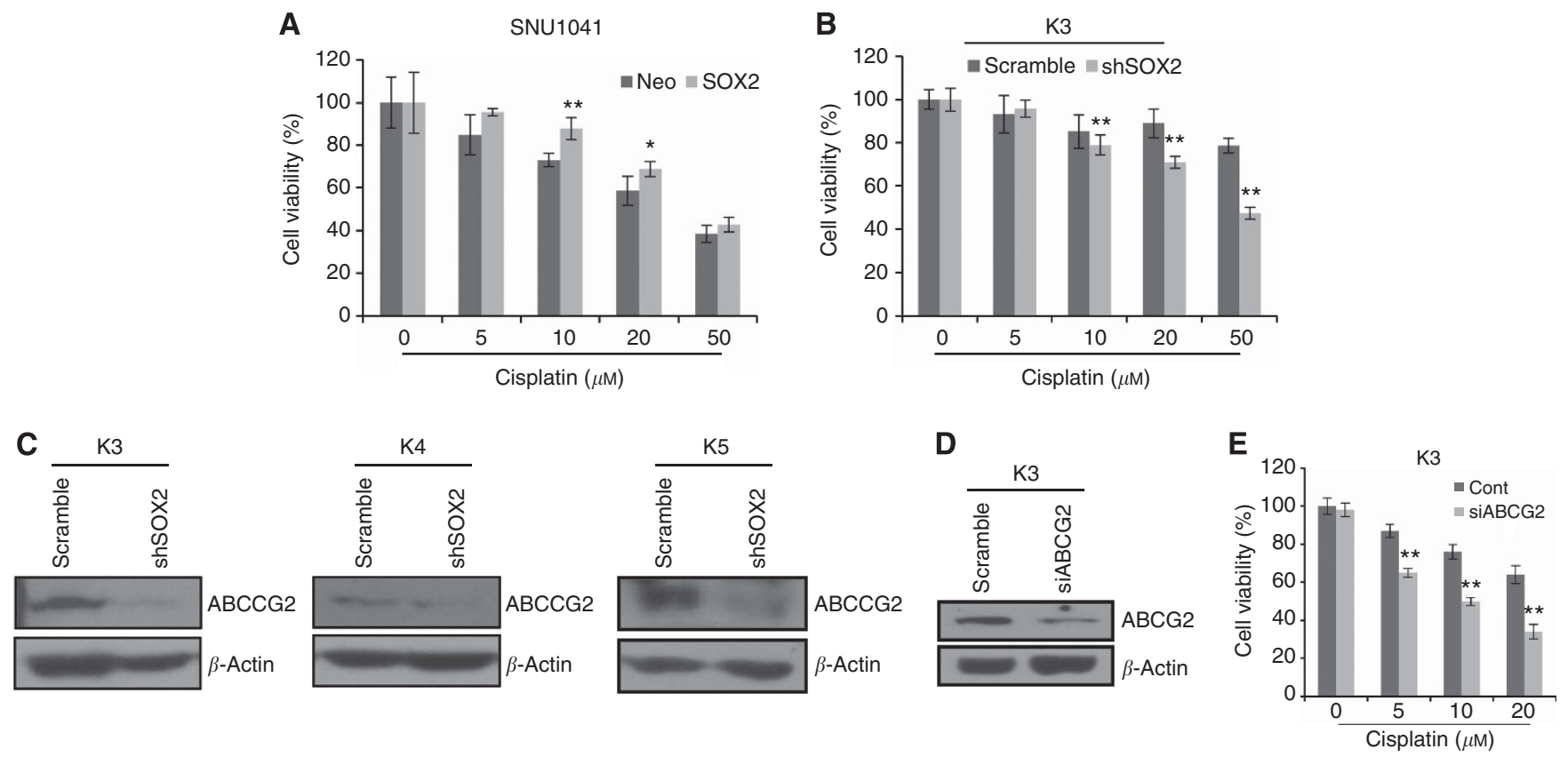

Figure 5. Suppression of SOX2 expression in primary HNSCC stem cells decreases antitumour drug resistance related to ABCG2. (A) Cell viability assay of cisplatin in control- and SOX2-overexpressing HNSCC cells. (B) Cell viability was determined after cisplatin treatment in control and shSOX2-transfected HNSCC stem-like cells. Treatments were performed in a dose-dependent manner (0-50 $\mu \mathrm{M}$, for $24 \mathrm{~h}, \mathrm{n}=3)$. (C) Expression levels of ABCG2 in HNSCC stem-like cells transfected with scrambled siRNA and in HNSCC stem-like cells transfected with shSOX2; confirmed by western blot analysis. (D) The protein level of ABCG2 after transfection of ABCG2-siRNA into HNSCC stem-like cells. (E) Cell viability determined after cisplatin treatment in HNSCC stem-like cells transfected with scrambled siRNA and in HNSCC stem-like cells transfected with siABCG2 $(n=3)$.
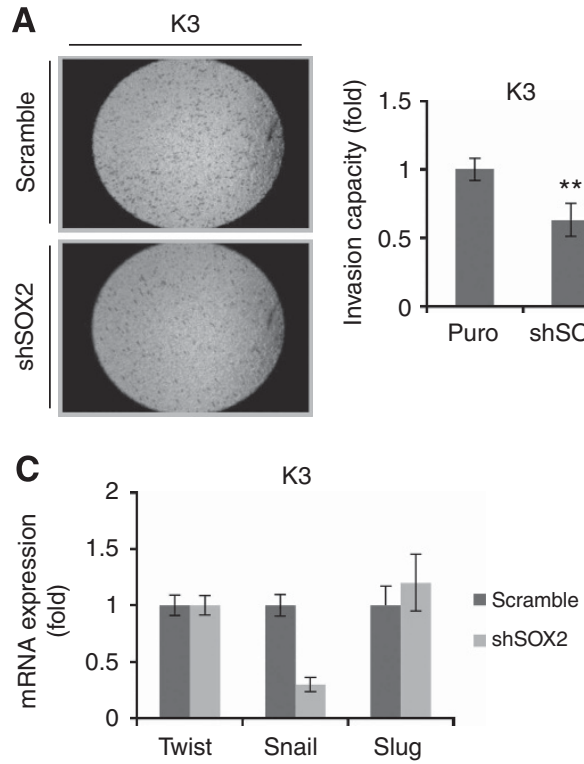

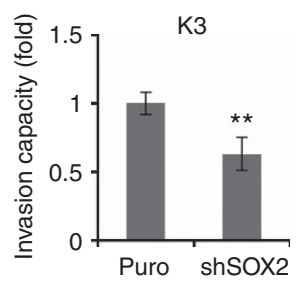

D
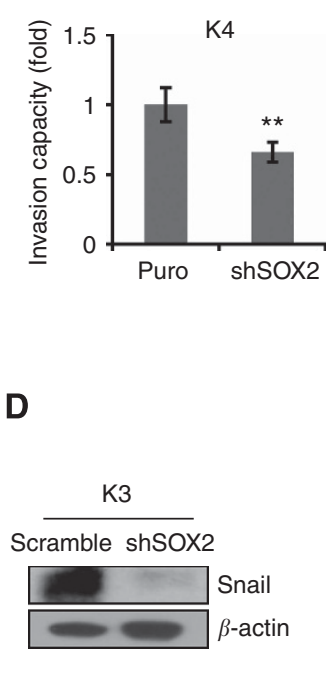

B
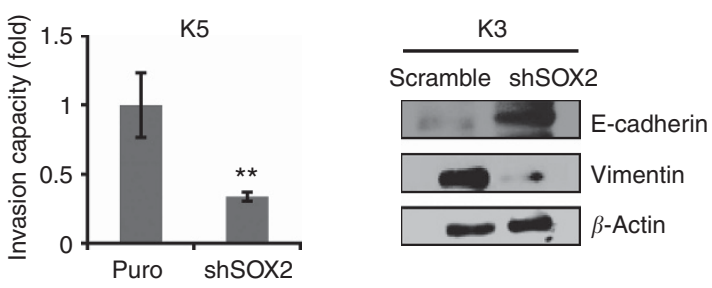

E
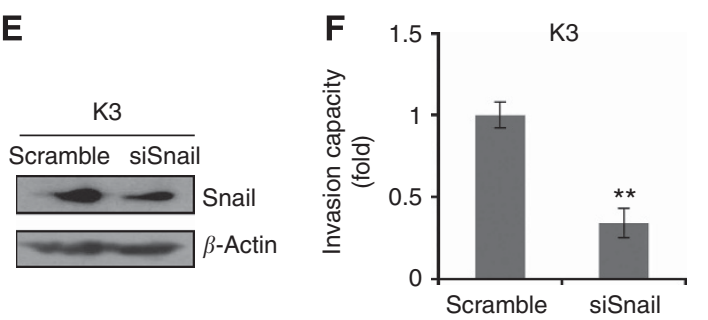

Figure 6. Suppression of the SOX2 expression level in primary HNSCC stem-like cells decreases tumour invasiveness that is related to SNAIL signalling. (A) The Transwell assay performed in HNSCC stem-like cells and HNSCC stem-like cells transfected with shSOX2 ( $n=3$ ). A representative picture shows the cells attached to the layer of the bottom plate (left). The graph shows the relative invasion capacity in HNSCC stem-like cells and in HNSCC stem-like cells transfected with shSOX2. (B) Western blot analysis using anti-vimentin and anti-E-cadherin antibodies in HNSCC stem-like cells and in HNSCC stem-like cells transfected with shSOX2. (C) RT-qPCR analysis of the expression of TWIST, SNAIL, and SLUG genes, performed in HNSCC stem-like cells and in HNSCC stem-like cells transfected with shSOX2. (D) SNAIL expression level confirmed using western blot analysis in HNSCC stem-like cells and in HNSCC stem-like cells transfected with shSOX2. (E) The SNAIL expression level confirmed using western blot analysis in HNSCC stem-like cells and in HNSCC stem-like cells transfected with siSnail. (F) The Transwell assay performed in HNSCC stem-like cells and in HNSCC stem-like cells transfected with siSnail $(n=3)$.

In the orthotopic tongue model, xenografted HNSCC CSCshSOX2 cells also exhibited reduced tumorigenicity compared with control HNSCC CSCs (Figure 7B). Additional analysis using an immunohistochemical assay with Ki-67 (Figure 7C) and CD44 (Figure 7D) revealed that the SOX2 knockdown decreased the proliferative ability and stemness properties in the xenografted 
A

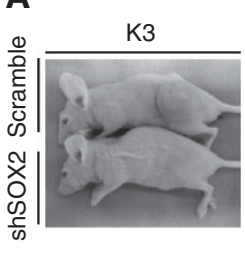

Injected cells Tumour formation

Scramble

$10^{4}$

$10^{5}$

shSOX2

$10^{5}$

K3
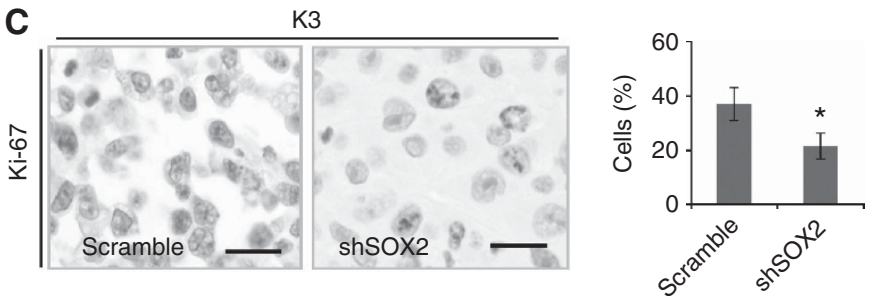

D

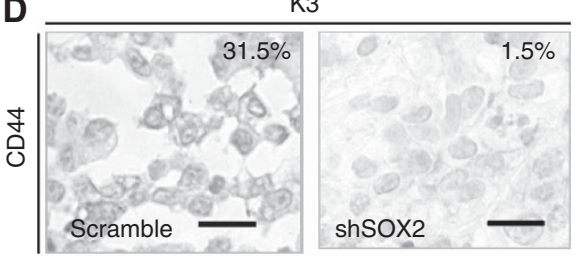

B $\mathrm{K} 3$

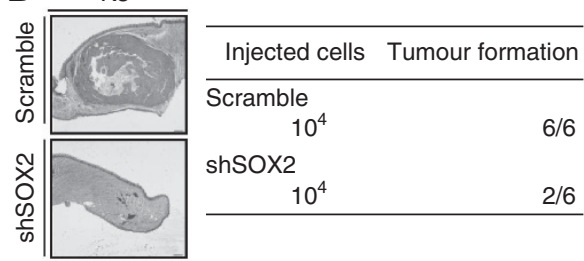

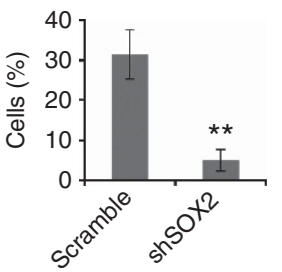

Figure 7. Suppression of the SOX2 expression level in primary HNSCC stem-like cells decreases tumorigenicity in vivo. (A) Representative tumour growth (left) and a summary of the subcutaneous tumour growth ability (right) of HNSCC stem-like cells and of HNSCC stem-like cells transfected with shSOX2. Groups of mice were inoculated with control-HNSCC stem-like cells and shSOX2- HNSCC stem-like cells at $10^{4}$ and $10^{5}$ cells per injection, respectively. Engrafted mice were inspected weekly for tumour development by visual observation and palpation until 8 weeks postinjection. In the orthotopic tongue experiment, control-HNSCC stem-like cells and shSOX2- HNSCC stem-like cells $\left(1 \times 10^{4}\right.$ per animal) were engrafted directly into the anterior tongue and the mice were then examined every other day for the development of tongue tumours. (B) Photos of tongues bearing HNSCC stem-like cells and HNSCC stem-like cells transfected with shSOX2 (left) and a summary of the orthotopic tumour growth capacity of HNSCC stem-like cells and of HNSCC stem-like cells transfected with shSOX2 (right). (C) Ki-67 antibody staining of the tumour tissue that originated from injected HNSCC stem-like cells or HNSCC stem-like cells transfected with shSOX2, according to immunohistochemical staining (left) and its quantification (right). Nuclei were stained with haematoxylin. (D) The anti-CD44 antibody staining of tumour tissue that originated from injected HNSCC stem-like cells or HNSCC stem-like cells transfected with shSOX2,according to immunohistochemical staining (left) and its quantification (right). Nuclei were stained with haematoxylin.

tumour tissue, which resulted from inoculation of mice with tumour cells. Overall, these data indicate that SOX2 regulates HNSCC tumorigenesis and maintenance in vivo.

\section{DISCUSSION}

HNSCC, as other solid tumours, is characterised by a histologically highly heterogeneous population of cancer cells that can be divided into populations with stem cell characteristics, called CSC, and more differentiated cell types. Previous studies by different groups have suggested that HNSCC CSCs are closely associated with aggressive growth, invasion, and metastasis, and with resistance to conventional chemo- and radiotherapy, resulting in tumour recurrence and regrowth after treatment (Begg, 2012; Zhang et al, 2012; Chen et al, 2013). Thus, targeted elimination of these CSCs could be a new promising therapeutic strategy against HNSCC. To this end, a systematic functional study regarding specific molecules regulating CSC proliferation, stemness, and chemoresistance has to be conducted.

Cancer stem cell, as implied by the term, is defined as a subset of tumour cells with stemness traits. They can be derived from transformation of normal stem cells, capable of self-renewal and generation of a heterogeneous progeny; CSCs can also be derived from differentiated cells that have acquired self-renewal properties as a consequence of genetic or epigenetic alterations (Kumar et al,
2012). Thus, CSCs may share common pathways that are essential for physiologic development of embryonic or adult stem cells (Nichols et al, 1998). For example, the $\mathrm{Wnt} / \beta$-catenin signalling may contribute to the regulation of stem cells/progenitor cells' selfrenewal in the skin epithelium of mammals (Clevers, 2006). On the other hand, mutations or deregulated expression of components of the Wnt signalling pathway can induce epithelial-origin cancer, such as colon cancer (Bertrand et al, 2012). In addition, transgenic mice suggest that activation of the Wnt signalling pathway in epidermal stem cells leads to epithelial cancers (Li et al, 2000). As mentioned above, SOX2 can also be involved in both embryonic development processes and in cancer initiation. SOX2 has a critical role in EGF receptor-mediated self-renewal of pancreatic CSCs (Rybak and Tang, 2013). SOX2 overexpression increases the proportion of breast CSC by activating the Wnt signalling pathway (Piva et al, 2014). However, the role of SOX2 in HNSCC remains unknown.

The present study showed that ectopic expression of SOX2 promotes cell proliferation through cyclin B1 activation and induces dedifferentiation of HNSCC cells. In addition, a knockdown of SOX2 led to significant inhibition of HNSCC CSC stemness-associated features, such as self-renewal capacity, stem cell expression markers, chemoresistance (through ABCG2 expression), and tumorigenesis, in a serial-dilution xenograft. Moreover, SOX2 increases invasion capacity, which is related to EMT modulation via snail expression. Finally, there was a significant 
correlation between SOX2 expression and survival of primary HNSCC patients. Overall, SOX2 is one of the master regulators of stemness in HNSCC CSCs.

Our study showed that SOX2 promotes HNSCC proliferation via cyclin $\mathrm{B} 1$ upregulation. SOX2 can regulate the cell cycle in pancreatic cancer cells through direct repression of the $p 21$ gene expression (Herreros-Villanueva et al, 2013). Furthermore, p21 directly binds to and inhibits the activity of Cdk1/cyclin B1 in colorectal cancer cells (Archer et al, 2005). Thus, SOX2 may directly upregulate cyclin B1 expression in HNSCC.

A few studies have reported that the $A B C$ transporter diminishes the sensitivity of cancer cells to anticancer drugs, such as cisplatin, in numerous HNSCC stem cells that originate from patients (Lim et al, 2011). Our findings suggest that SOX2 may be a bona fide cancer stemness factor that is associated with chemoresistance via the ABCG2 pathway. This is because their expression was found to be elevated in HNSCC patients and governs many CSC features in vitro and in vivo.

Previous studies have shown an association between the CSC phenotype and invasiveness, along with EMT (Liu et al, 2013; White et al, 2013). Our study showed that SOX2 knockdown reduces the invasion capacity of CSC through the EMT process. Several studies presented evidence of association between SOX2 expression and the EMT process. A knockdown of SOX2 in colorectal cancer SW620 cells induced the mesenchymal-epithelial transition process with recognised changes in the expression of key genes involved in the EMT process, including E-cadherin and vimentin (Han et al, 2012). SOX2 overexpression drives a cancer cell's dedifferentiation from epithelial to an EMT-like phenotype in pancreatic cancer cells. Specifically, our study showed that SOX2 expression may be linked to SNAIL expression, which is known to drive EMT, as an underlying mechanism of SOX2-mediated invasiveness and the broad capacity of HNSCC CSC. Using a ChIP assay (chromatin immunoprecipitation), Herreros-Villanueva et al (2013) confirmed that SOX2 can directly bind to and regulate the SNAIL gene involved in EMT in pancreatic cancer cells. Taken together, SOX2 and SNAIL can be the key molecules mediating invasive traits shared by HNSCC CSC and EMT.

In summary, our findings revealed that SOX2 can cause cancer cells to express CSC features and performs a crucial function in the maintenance of cancer stemness in HNSCC stem-like cells derived from patients. In addition, SOX2 has prognostic value in the evaluation of HNSCC patients. Given the importance of SOX2 in HNSCC, our findings not only provide an improved understanding of the molecular mechanism of maintenance of HNSCC stemness but also suggest possible therapeutic targets.

\section{ACKNOWLEDGEMENTS}

This work was supported by the Samsung Biomedical Research Institute grant (Grant Number: SBRI GL1B32611 to SH Lee) and the Korea government (MEST) (Grant Number: 2012R1A2A2A 01046214 to YC Lim).

\section{CONFLICT OF INTEREST}

The authors declare no conflict of interest.

\section{REFERENCES}

Albers AE, Chen C, Köberle B, Qian X, Klussmann JP, Wollenberg B,

Kaufmann AM (2012) Stem cells in squamous head and neck cancer. Crit Rev Oncol Hematol 81: 224-240.
Archer SY, Johnson J, Kim HJ, Ma Q, Mou H, Daesety V, Meng S, Hodin RA (2005) The histone deacetylase inhibitor butyrate downregulates cyclin B1 gene expression via a p21/WAF-1-dependent mechanism in human colon cancer cells. Am J Physiol Gastrointest Liver Physiol 289: G696-G703.

Bass AJ, Watanabe H, Mermel CH, Yu S, Perner S, Verhaak RG, Kim SY, Wardwell L, Tamayo P, Gat-Viks I, Ramos AH, Woo MS, Weir BA, Getz G, Beroukhim R, O’Kelly M, Dutt A, Rozenblatt-Rosen O, Dziunycz P, Komisarof J, Chirieac LR, Lafargue CJ, Scheble V, Wilbertz T, Ma C, Rao S, Nakagawa H, Stairs DB, Lin L, Giordano TJ, Wagner P, Minna JD, Gazdar AF, Zhu CQ, Brose MS, Cecconello I, Jr UR, Marie SK, Dahl O, Shivdasani RA, Tsao MS, Rubin MA, Wong KK, Regev A, Hahn WC, Beer DG, Rustgi AK, Meyerson M (2009) SOX2 is an amplified lineage-survival oncogene in lung and esophageal squamous cell carcinomas. Nat Genet 41: 1238-1242.

Begg AC (2012) Predicting recurrence after radiotherapy in head and neck cancer. Semin Radiat Oncol 22: 108-118.

Bertrand FE, Augus CW, Partis WJ, Sigounas G (2012) Developmental pathways in colon cancer: crosstalk between WNT, BMP, Hedgehog and Notch. Cell Cycle 11: 4344-4351.

Carvalho AL, Nishimoto IN, Califano JA, Kowalski LP (2005) Trends in incidence and prognosis for head and neck cancer in the United States: a site-specific analysis of the SEER database. Int J Cancer 114: 806-816.

Chen C, Zimmermann M, Tinhofer I, Kaufmann AM, Albers AE (2013) Epithelial-to-mesenchymal transition and cancer stem(-like) cells in head and neck squamous cell carcinoma. Cancer Lett 338: 47-56.

Chen KG, Sikic BI (2012) Molecular pathways: regulation and therapeutic implications of multidrug resistance. Clin Cancer Res 18: 1863-1869.

Chen S, Xu Y, Chen Y, Li X, Mou W, Wang L, Liu Y, Reisfeld RA, Xiang R, Lv D, Li N (2012) SOX2 gene regulates the transcriptional network of oncogenes and affects tumorigenesis of human lung cancer cells. PLoS One 7: e36326.

Clevers H (2006) Wnt/beta-catenin signalling in development and disease. Cell 127: 469-480.

Du L, Yang Y, Xiao X, Wang C, Zhang X, Wang L, Zhang X, Li W, Zheng G, Wang S, Dong Z (2011) SOX2 nuclear expression is closely associated with poor prognosis in patients with histologically node-negative oral tongue squamous cell carcinoma. Oral Oncol 47: 709-713.

Han X, Fang X, Lou X, Hua D, Ding W, Foltz G, Hood L, Yuan Y, Lin B (2012) Silencing SOX2 induced mesenchymal-epithelial transition and its expression predicts liver and lymph node metastasis of CRC patients. PLoS One 7: e41335.

Herreros-Villanueva M, Zhang JS, Koenig A, Abel EV, Smyrk TC, Bamlet WR, de Narvajas AA, Gomez TS, Simeone DM, Bujanda L, Billadeau DD (2013) SOX2 promotes dedifferentiation and imparts stem cell-like features to pancreatic cancer cells. Oncogenesis 2: e61.

Howell GM, Grandis JR (2005) Molecular mediators of metastasis in head and neck squamous cell carcinoma. Head Neck 27: 710-717.

Ikushima H, Todo T, Ino Y, Takahashi M, Miyazawa K, Miyazono K (2009) Autocrine TGF-beta signalling maintains tumorigenicity of glioma-initiating cells through Sry-related HMG-box factors. Cell Stem Cell 5: 504-514.

Jemal A, Murray T, Samuels A, Ghafoor A, Ward E, Thun MJ (2003) Cancer statistics, 2003. CA Cancer J Clin 53: 5-26.

Keramari M, Razavi J, Ingman KA, Patsch C, Edenhofer F, Ward CM, Kimber SJ (2010) SOX2 is essential for formation of trophectoderm in the preimplantation embryo. PLoS One 5: e13952.

Kumar SM, Liu S, Lu H, Zhang H, Zhang PJ, Gimotty PA, Guerra M, Guo W, $\mathrm{Xu}$ X (2012) Acquired cancer stem cell phenotypes through Oct4mediated dedifferentiation. Oncogene 31: 4898-4911.

Lai SY, Childs EE, Xi S, Coppelli FM, Gooding WE, Wells A, Ferris RL, Grandis JR (2005) Erythropoietin-mediated activation of JAK-STAT signaling contributes to cellular invasion in head and neck squamous cell carcinoma. Oncogene 24: 4442-4449.

Leis O, Eguiara A, Lopez-Arribillaga E, Alberdi MJ, Hernandez-Garcia S, Elorriaga K, Pandiella A, Rezola R, Martin AG (2012) SOX2 expression in breast tumours and activation in breast cancer stem cells. Oncogene 31: $1354-1365$.

Li Y, Hively WP, Varmus HE (2000) Use of MMTV-Wnt-1 transgenic mice for studying the genetic basis of breast cancer. Oncogene 19: 1002-1009.

Lim YC, Oh SY, Cha YY, Kim SH, Jin X, Kim H (2011) Cancer stem cell traits in squamospheres derived from primary head and neck squamous cell carcinomas. Oral Oncol 47: 83-91. 
Liu J, Ruan B, You N, Huang Q, Liu W, Dang Z, Xu W, Zhou T, Ji R, Cao Y, Li X, Wang D, Tao K, Dou K (2013) Downregulation of miR-200a induces EMT phenotypes and CSC-like signatures through targeting the $\beta$-catenin pathway in hepatic oval cells. PLoS One 8: e79409.

Mani SA, Guo W, Liao MJ, Eaton EN, Ayyanan A, Zhou AY, Brooks M, Reinhard F, Zhang CC, Shipitsin M, Campbell LL, Polyak K, Brisken C, Yang J, Weinberg RA (2008) The epithelial-mesenchymal transition generates cells with properties of stem cells. Cell 133: 704-715.

Nichols J, Zevnik B, Anastassiadis K, Niwa H, Klewe-Nebenius D, Chambers I, Schöler H, Smith A Nichols J (1998) Formation of pluripotent stem cells in the mammalian embryo depends. Cell 95: 379-391.

Okubo T, Clark C, Hogan BL (2009) Cell lineage mapping of taste bud cells and keratinocytes in the mouse tongue and soft palate. Stem Cells 27: $442-450$.

Piva M, Domenici G, Iriondo O, Rábano M, Simões BM, Comaills V, Barredo I, López-Ruiz JA, Zabalza I, Kypta R, Vivanco Md (2014) SOX2 promotes tamoxifen resistance in breast cancer cells. EMBO Mol Med 6: 66-79.

Rybak AP, Tang D (2013) SOX2 plays a critical role in EGFR-mediated self-renewal of human prostate cancer stem-like cells. Cell Signal 25: 2734-2742.

Sarkar A, Hochedlinger K (2013) The SOX family of transcription factors: versatile regulators of stem and progenitor cell fate. Cell Stem Cel 12: $15-30$.

Schröck A, Bode M, Göke FJ, Bareiss PM, Schairer R, Wang H, Weichert W, Franzen A, Kirsten R, van Bremen T, Queisser A, Kristiansen G, Heasley L, Bootz F, Lengerke C, Perner S (2014) Expression and role of the embryonic protein SOX2 in head and neck squamous cell carcinoma. Carcinogenesis 35: 1636-1642.

Schröck A, Göke F, Wagner P, Bode M, Franzen A, Braun M, Huss S, Agaimy A, Ihrler S, Menon R, Kirsten R, Kristiansen G, Bootz F, Lengerke C, Perner S (2013) Sex determining region Y-box 2 (SOX2) amplification is an independent indicator of disease recurrence in sinonasal cancer. PLoS One 8: e59201.

Tang XB, Shen XH, Li L, Zhang YF, Chen GQ (2013) SOX2 overexpression correlates with poor prognosis in laryngeal squamous cell carcinoma. Auris Nasus Larynx 40: 481-486.

White RA, Neiman JM, Reddi A, Han G, Birlea S, Mitra D, Dionne L, Fernandez P, Murao K, Bian L, Keysar SB, Goldstein NB, Song N, Bornstein S, Han Z, Lu X, Wisell J, Li F, Song J, Lu SL, Jimeno A, Roop DR, Wang XJ (2013) Epithelial stem cell mutations that promote squamous cell carcinoma metastasis. J Clin Invest 123: 4390-4404.

Yamano Y, Uzawa K, Saito K, Nakashima D, Kasamatsu A, Koike H, Kouzu Y, Shinozuka K, Nakatani K, Negoro K, Fujita S, Tanzawa H (2010) Identification of cisplatin-resistance related genes in head and neck squamous cell carcinoma. Int J Cancer 126: 437-449.

Yu F, Sim AC, Li C, Li Y, Zhao X, Wang DY, Loh KS (2013) Identification of a subpopulation of nasopharyngeal carcinoma cells with cancer stemlike cell properties by high aldehyde dehydrogenase activity. Laryngoscope 123: 1903-1911.

Zhang S, Balch C, Chan MW, Lai HC, Matei D, Schilder JM, Yan PS, Huang TH, Nephew KP (2008) Identification and characterization of ovarian cancer-initiating cells from primary human tumors. Cancer Res 68: 4311-4320.

Zhang Z, Filho MS, Nor JE (2012) The biology of head and neck cancer stem cells. Oral Oncol 48: 1-9.

This work is published under the standard license to publish agreement. After 12 months the work will become freely available and the license terms will switch to a Creative Commons AttributionNonCommercial-Share Alike 3.0 Unported License.

Supplementary Information accompanies this paper on British Journal of Cancer website (http://www.nature.com/bjc) 